\title{
Anti-theft Fingerprint Security System for Motorcycles Using Arduino UNO, GPS/GSM Module
}

\author{
Kiran CS* \\ Department of Electrical and Electronics Engineering, Vidyavardhaka College of Engineering, \\ Mysore, Karnataka 570002. India; cskiran98@gmail.com
}

\begin{abstract}
Objectives: To develop biometric based suitable vehicle security system. Anti-theft fingerprint security system is one of the advanced security systems. Methods: The fingerprint security system is installed on the bike; using the Global System Mobile Communication (GSM)/Global Positioning System (GPS) modules, the owner can interact with the bike and can track the real time location of his bike. The parts which are used for the implementation of the hardware modules are as follows: Arduino microcontroller UNO, fingerprint sensor, and four channel relay module. Findings: In this study, a unique design has been proposed to increase the security system for the vehicles by using fingerprint sensors. By using the GSM/ GPS modem, the real time location of the vehicle can be obtained and the ignition state of the vehicle can also be controlled by the users' smartphone. So, this will be the cheapest and very efficient mode of security system for the motorcycle vehicles.
\end{abstract}

Keywords: Fingerprint Sensor, GSM/GPS Technology, Arduino UNO, Vehicle Tracking System.

\section{Introduction}

Nowadays, even the cheaper smartphones will be having the fingerprint sensors for the purpose of security. 1 But till today no bikes in the market are having the fingerprint security system, although the theft of vehicles are being increased day by day. The data show that the motor vehicle theft remains the least-solved crime in India. A total of 44,158 cases of vehicle theft were reported in 2018 out of which only $19.6 \%$ cases were solved. But it is amazing to know how a simple idea helps in changing the whole system by providing a modern security system for the vehicles.

So, in this study, a unique design has been proposed to increase the security system for the vehicles by using fingerprint sensors. This is the extension in the field of the automatic controlling of the vehicles. By this design, a fingerprint sensor for a bike has been designed economically. The Global System Mobile Communication (GSM)/Global Positioning System (GPS) module has also been used to track the position of the vehicle in case of theft. $\underline{2}$
GPS/GSM system is the advanced system which allows the user to track the vehicle using a GPS and with the GSM module. $\underline{\underline{3}-6}$ Therefore, by using this system the user can obtain the location information of his motorcycle vehicle where it is currently, on his smartphone and he can track it on the google maps. ${ }^{-}-\underline{9}$ The GSM/GPS module is powered by a microcontroller which is programmed using Arduino. The main motto of this research study is to provide security for vehicles using fingerprint system. If anyone else other than the user enters the unregistered fingerprint, the user will be notified using the GSM modem. Now the owner will be alerted and he can track his vehicle using his smartphone with the help of GPS. And the engine could also be turned off using the GSM by sending commands to the vehicle.

\section{Experimental Design}

In this study, an antitheft security system using fingerprint module technology has been discussed. The GSM/GPS module is also used for the tracking of devices and also to

${ }^{*}$ Author for correspondence 
send and receive messages from vehicles to the user and vice versa.

A fingerprint hardware module is implemented to unlock the system using the authorised users only. This technology prevents the theft of vehicles. $\frac{10}{}$ And also the attempt to steal the vehicle will also be notified to the user using GSM technology.

The proposed design ...., the proposed design uses "GPS" and "GSM". GPS helps in tracking the vehicle location in real time, and GSM helps in sending these data to the user when there is a need. In case of theft, the user can send the command to the arduino unit and then the arduinouno issue the control command to stop the ignition of the vehicle.

\section{Electronic Components}

In this project, the parts which are used for the implementation of the hardware modules are Arduino microcontroller UNO, fingerprint sensor, and four channel relay module are shown in Figures 1-3.

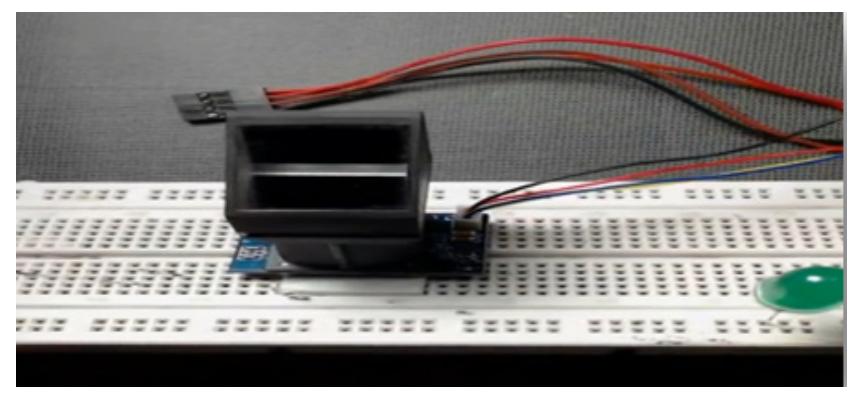

Figure 1. Fingerprint sensor R305.

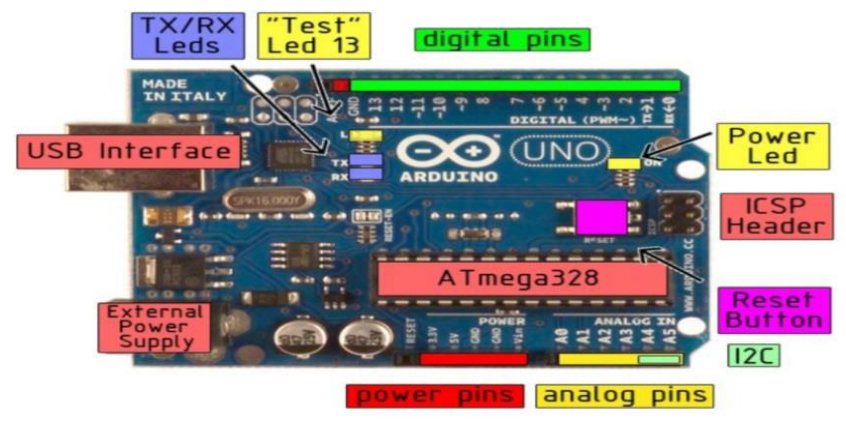

Arduino microcontroller UNO:

Figure 2. Arduino UNO.

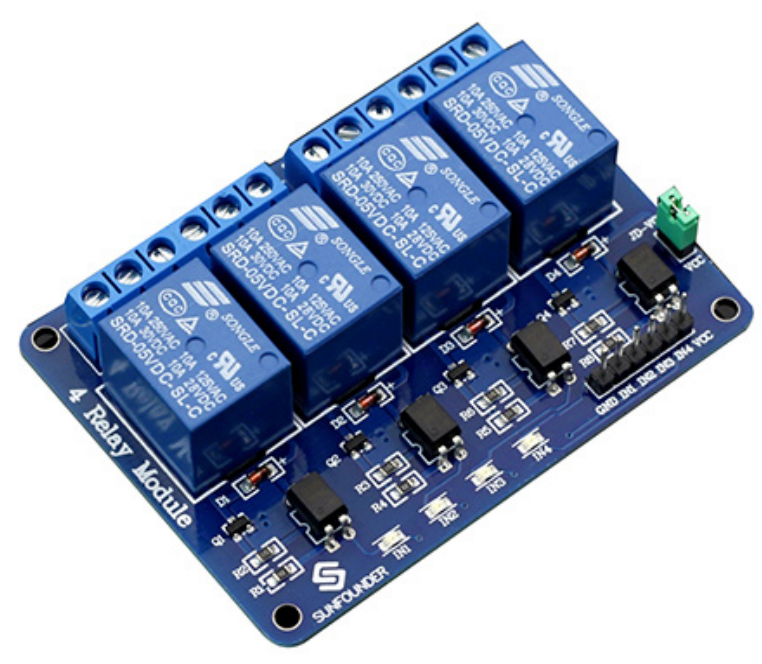

Figure 3. Four channel relay.

\subsection{R305 Fingerprint Sensor Module}

It is device which identifies the fingerprint of a user in order to grant or deny permission to access the system. It has four terminals VCC, Ground, Transmit (Tx) pin, and Receive $(\mathrm{Rx})$ pin. The user can store the fingerprint data in this module and can configure it in 1:N mode for identifying the person. It requires a DC power supply of $3.6 \mathrm{~V}$.

It is a microcontroller board based on the ATmega328. It has 14 digital input and output pins. And it consists of 6 analogiputs, USB interface, reset button, power led. It requires external power supply to power on. It also has transmit $(\mathrm{Tx})$ and receive $(\mathrm{Rx})$ leds.

\subsection{Four Channel Relay}

Relay is an electromagnetic device that acts as a switch to control various circuits by one signal. It also separates two circuits electrically and connects them magnetically. In this study, a junction box has been used for the implementation of the developed design. Various devices are connected to the junction box and supplied with the $\mathrm{AC}$ power.

\subsection{GPS/GSM}

The GSM module is used as a communication link between the user and the vehicle security system. The GSM module sends data to the user. In case of theft, the GPS module collects the location of the vehicle and stores it in the microcontroller and then the GSM module collects data from the microcontroller and sends the data 
to the user. Now the user can locate his vehicle using google maps. And also the user can send command to the microcontroller to stop the flow of fuel and switch of the ignition using GSM module.

\section{Proposed Architecture}

\subsection{Hardware Section}

The circuit connections are made as shown in the circuit diagram (Figure 4) using the breadboard, R305 fingerprint sensor.

The R305 fingerprint sensor module has four pins Vcc, Ground, Transmit (Tx) pin, and Receive ( $\mathrm{Rx}$ ) pin. Tx pin is connected to the pin 3 of the arduino, and the $\mathrm{Rx}$ pin is connected to the pin 2 of the arduino module. Vcc is supplied with 3.3V voltage and the Gnd pin is grounded.

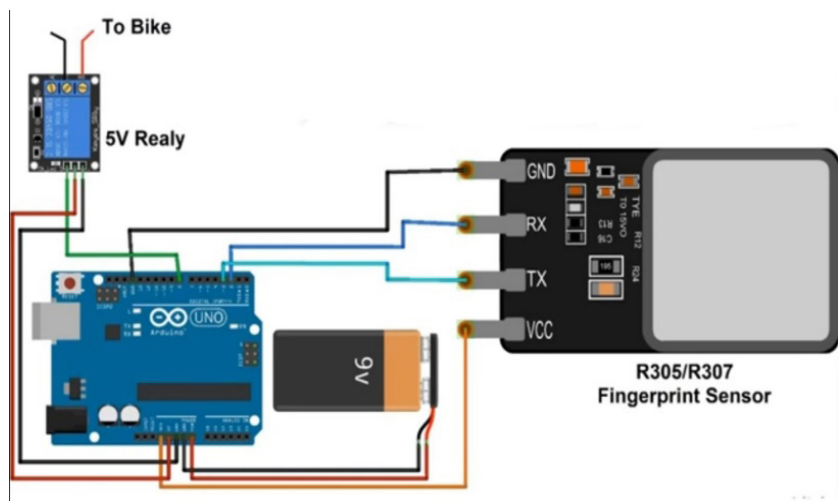

Figure 4. Circuit diagram of antitheft fingerprint secrity system.

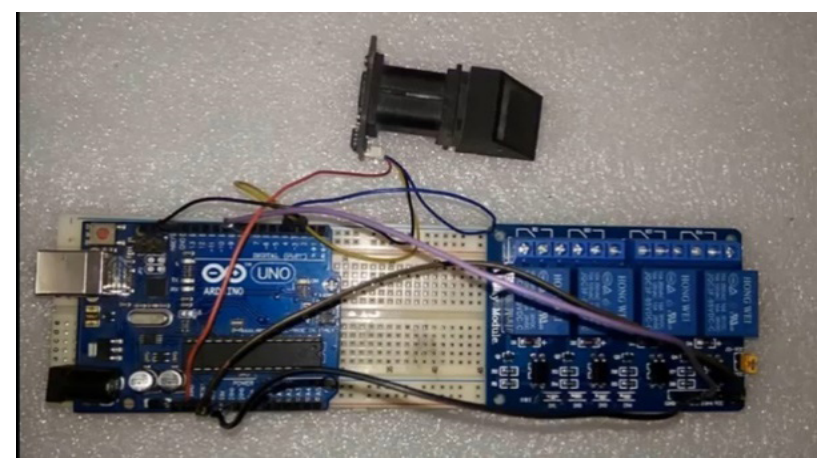

Figure 5. Circuit connections of the fingerprint security system.
In this design, four channel relay has been used. But a single channel relay could also be used instead of this. Connect the input pin i.e. pin 8 of arduino UNO and supply it with $5 \mathrm{~V}$ power. Now the circuit connection has been completed.

And the circuit connections can be seen in Figure 5. After the hardware connections of the module has been made, the coding must be done to enroll the fingerprint.

\subsection{Programming Section}

Now obtain an arduino ID and create a library in this project 2 sets of coding has been done

- Code for enrolling the fingerprint

- Code for matching the fingerprint

Code for enrolling is used to store fingerprint in fingerprint module. Actually, the fingerprint is stored in fingerprint module but not in the arduino. So, this program helps in enrolling the fingerprint in the fingerprint module. Now the compiling process takes place in the software. After this, the fingerprint has to be registered. Now by going to the serial monitor on top right side of the monitor a new window will be opened and we need to enter ID from 1 to 127. Any of them may be used and the fingerprint will be stored in the opened ID. And now it will ask us to enter the fingerprint. After placing the finger on the fingerprint module, the image will be taken and it will be stored in the Arduino. Multiple fingerprints can be registered.

Now programming for starting the bike must be done using the relay module. In this program, the pin 8 has been defined as the output and the relay output has been connected to the bike. So, when the registered fingerprint is placed on the fingerprint sensor the valve gets opened and petrol starts flowing and the bike gets started. If in case fingerprint does not match then the valve remains closed and the petrol will never flow and the bike does not get started. Now upload this code to the Arduino UNO board directly and once the uploading is done, install the hardware module on the bike by replacing the self-start button with this anti-theft fingerprint security system as shown in Figure 6.

And using GSM/GPS module the security of the bike can be increased so that in the case of theft the real time location of the vehicle can be tracked using the GPS module. This GPS system collects the location of the vehicle and will be storing the data in the Arduino 


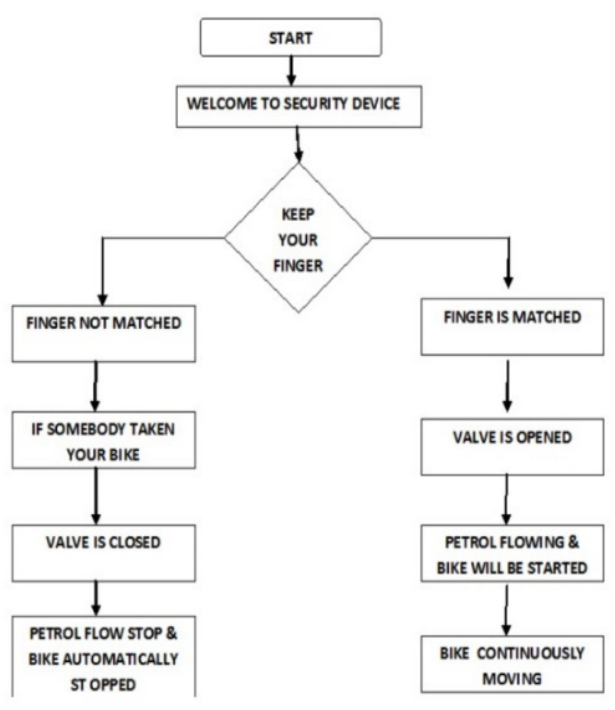

Figure 6. Security flowchart.

at certain time intervals. At the time of theft, the GSM can be used to obtain this stored location data. The user obtains the real time location of the vehicle using GSM module.

Figure 7 shows the alert message sent to the user of the vehicle in case of emergencies using GSM technology. The vehicle will be having GPS receiver which helps the user to obtain the real time location of the vehicle. The GSM modem notifies the user with the data. These data consist of the vehicle location, longitude, latitude, altitude of the location. And now by using these data, we can locate the exact location of the vehicle using Google Maps. This

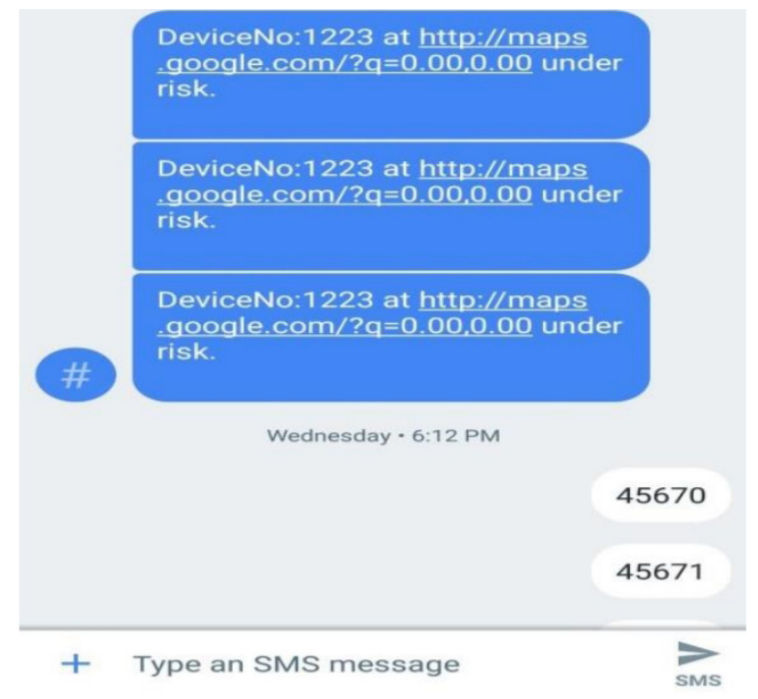

Figure 7. SMS alert sent to the user's smartphone. system not only notifies the user with the location of the vehicle but also helps the user to control the ignition of the vehicle using his smartphone. The user can easily deactivate his vehicle's engine as soon as he comes to know about the theft of his vehicle. When the user sends the command through GSM to the Arduino, the valve gets closed and the flow of fuel gets stopped and then the vehicle also stops.

So, this design not only helps the user to unlock his system without keys but also tightens the security system and provides multilayer protection to the vehicle. The fingerprint sensor installed on the bike has been shown in Figure 8.

\section{Advantages}

- The proposed model is economical and is highly efficient.

- The proposed model is user friendly.

- This model provides the better and extension in the automatic control of the vehicles.

- This system helps to avoid theft of vehicles by providing anti-theft security to the system.

- The system is flexible by using the Arduino Uno model.

\section{Conclusion}

The proposed fingerprint, GPS/GSM-based vehicle security system is the modern and reliable technique of security mechanism. The system which is developed by

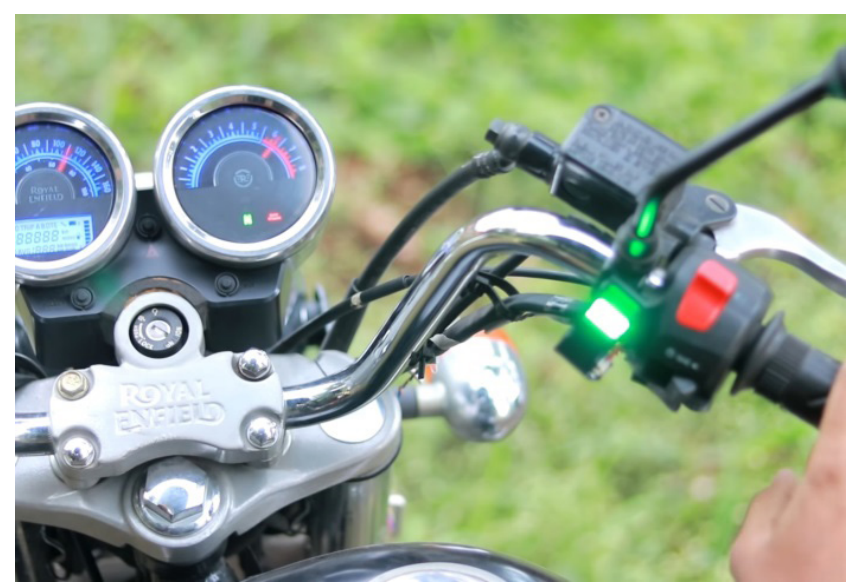

Figure 8. Fingerprint sensor installed on the bike. 
this project provides commodious protection against theft. The fingerprint sensor basically removes the use of key to start the ignition of the bike and it removes the threat of theft by providing the additional layer of security to the vehicle. The proposed model is highly economical in cost and it is very user friendly. And by using the GSM/ GPS modem, the real time location of the vehicle can be obtained and the ignition state of the vehicle can also be controlled by the users' smartphone.

\section{Acknowledgement}

The authors express gratitude to Accendere Knowledge Management Services Pvt Ltd for the assistance provided in preparing the manuscript.

\section{References}

1. Wimberly $\mathrm{H}$, Liebrock LM. Using fingerprint authentication to system security; an empirical study. In: IEEE symposium on security and privacy; 2011. P. 1-9.

2. Hu JM, Li J, Li GH. Automobile anti-theft system based on GSM and GPS module. In: 2012 fifth international conference on intelligent networks and intelligent systems (ICINIS); 2012. P. 199-201.
3. Soaid MF, Kamaludin MA, Megat Ali MSA. Vehicle location finder using global position system \& global system for mobile. In: Control \& system graduate research colloquium (ICSGRC); 2014. P. 279-84

4. Verma P, Bhatia JS. Design and development of GPS-GSM based tracking system with googlemap based monitoring. Int J Comput Sci Eng Appl (IJCSEA). 2013;3(3):1-12.

5. Singh P, Sethi T, Biswal BB, Pattanayak SK. A smart antitheft system for vehicle security. Int J Mater Mech Manuf. 2015;3(4):1-15.

6. Alshamsi H, Këpuska V, Alshamsi H. Real time vehicle tracking using Arduino mega. Int J Sci Technol. 2016;5(12):1-4

7. Nalina V, Sandesh AS, Sequiera RD, Jayarekha P. Cloud based multiple vehicle tracking and locking system. In: 2015 IEEE international advance computing conference (IACC); 2015. P. 1-6.

8. Kamble KP. Smart vehicle tracking system. Int J Distrib Parall Syst (IJDPS). 2012;3(4):1-10.

9. Abha Damani, Hardik Shah, Krishna Shah, Manish Vala. Global Positioning System for Object Tracking. Int J Comput Appl. 2015;109(8):40-45.

10. Dey M, Arif A, Mahmud A. Anti-theft protection of vehicle by GSM \& GPS with fingerprint verification. In: International conference on electrical, computer and communication engineering (ECCE); 2017. P. 1-5. 